\title{
Diferentes índices volumétricos dos pulmões de fetos com hérnia diafragmática congênita avaliados pela ultrassonografia tridimensional*
}

\author{
Different volumetric indices of lungs in fetuses \\ with congenital diaphragmatic hernia by \\ three-dimensional ultrasonography
}

\author{
Eduardo Takashi', Rodrigo Ruano², Marcelo Zugaib ${ }^{3}$
}

Takashi E, Ruano R, Zugaib M. Diferentes índices volumétricos dos pulmões de fetos com hérnia diafragmática congênita avaliados pela ultrassonografia tridimensional. Rev Med (São Paulo). 2009 jan.-mar.;88(1):58-65.

\begin{abstract}
RESUMO: Objetivo: avaliar o potencial das diferentes índices volumétricos de pulmões de fetos com hérnia diafragmática congênita isolada (HDC) medidos pela ultrassonografia tridimensional (US-3D) para predizer o resultado perinatal. Forma de estudo: Cinquenta e cinco fetos apresentando HDC foram avaliados prospectivamente pela US-3D entre janeiro de 2002 e maio de 2007. Os volumes pulmonares foram calculados pelo método rotacional na ultrassonografia tridimensional nos fetos com HDC (volumes observados) e comparados com aqueles descritos previamente em fetos normais (volumes esperados para determinada idade gestacional). As relações entre volumes pulmonares observados/esperados total (VPT), contralateral (VPC) e ipsilateral (VPI) foram calculadas, assim como a relação entre o volume pulmonar fetal total e o peso fetal estimado (VP/PF). Estas relações pulmonares foram comparadas com os resultados perinatais. Resultados: Óbitos perinatais ocorreram em 31 das 55 crianças $(56,4 \%)$, HP grave em 37 de 55 lactentes (67,3\%) e de HAP em 38 de 54 (excluindo uma morte no útero) de neonatos (70,4\%). HP grave e HAP ocorreram simultaneamente em $35(63,0 \%)$ de 54 crianças. Boas correlações entre todas as relações volumétricas do pulmão foram observadas; as relações VPT, VPC e VP/PF correlacionaram estatisticamente com óbitos perintais; VPT, VPI e VP/PF com o diagnóstico de HP grave; enquanto apenas VPT correlacionou-se com o diagnóstico pós-natal de HAP. As precisões do VPT, VPC, VPI e VP/PF em predizer óbitos perinatais foram $83,6 \%, 72,7 \%, 67,3 \%$ e $76,4 \%$, respectivamente. Conclusão: O VPT medido pelos US-3D apresenta maior precisão em predizer óbito perinatal, uma vez que está associado ao diagnóstico pós-natal de HP e HAP.
\end{abstract}

DESCRITORES: Hérnia diafragmática/congênito. Imagem tridimensional. Diagnóstico pré-natal. Pneumopatias/congênito. Pulmão/anormalidades. Ultra-sonografia pré-natal.

\footnotetext{
* Trabalho premiado no COMU - 2008. Área Clínica - Prêmio Oswaldo Cruz, 2008

1. Acadêmico da Faculdade de Medicina da Universidade de São Paulo (FMUSP).

2. Professor Doutor do Departamento de Obstetrícia e Ginecologia da FMUSP.

3. Professor Titular do Departamento de Obstetrícia e Ginecologia da FMUSP.
}

Endereço para Correspondência: Eduardo Takashi. Departamento de Obstetrícia e Ginecologia - Instituto Central Hospital das Clínicas da Faculdade de Medicina da Universidade de São Paulo. Av. Dr. Enéas de Carvalho Aguiar, 255

- ICHC 10andar. São Paulo, SP. E-mail: eduardotakashi@usp.br 


\section{INTRODUÇÃO}

A hérnia diafragmática congênita (HDC) ocorre em aproximadamente $1 \mathrm{em}$ cada 2200 nascidos vivos e está associado a uma taxa global de mortalidade neonatal de $50 \%$ nos casos com diagnóstico pré-natal' ${ }^{1}$. Durante a avaliação pré-natal , predizer o prognóstico perinatal continua sendo um grande desafio, apesar de ser fundamental para orientar cuidados neonatais imediatos além de ser importante para seleção de candidatos para as intervenções terapêuticas intra-uterinas, como a oclusão intermitente traqueal por fetoscopia ${ }^{1,2}$.

A mortalidade neonatal na HDC isolada tem sido diretamente relacionada com hipoplasia pulmonar grave $(\mathrm{PH})$. Avaliação pré-natal dos tamanhos estimados do pulmão pela ultrassonografia e ressonância magnética tem sido sugerida para predizer prognóstico ${ }^{3,9}$. Na relação pulmão/cabeça, a razão entre superfície do pulmão contralateral e a circunferência da cabeça em milímetros é medida por ultrassonografia bidimensional (US-2D) e é amplamente utilizada para este fim $^{7,8}$. Estudos recentes têm demonstrado que o volume pulmonar fetal pode ser estimado em casos de HDC utilizando ressonância magnética (MRI) ou ultrassonografia tridimensional (US-3D) $)^{3,9}$. Estudos iniciais sugerem que o volume pulmonar fetal total deve ser calculado pela soma dos volumes pulmonares direita e esquerda, a fim de predizer morte neonatal ${ }^{3,9}$. Estimativa do volume pulmonar fetal total na HDC pode ser realizada com precisão ${ }^{10}$; no entanto, a medida do volume pulmonar contralateral parece mais fácil do que medir o volume pulmonar ipsilateral. Além disso, nos casos de HDC, o pulmão fetal ipsilateral, geralmente, é mais hipoplásico do que o pulmão contralateral ${ }^{10}$. Até hoje, o potencial dos volumes dos pulmões ipsilateral e contralateral para a predizer o desfecho neonatal em fetos com HDC isolada nunca foi avaliada ou comparada um ao outro.

Mais recentemente, Tanigaki et al. ${ }^{11}$ propôs a utilização de uma relação entre o volume pulmonar fetal medido por ressonância nuclear magnética e o peso corporal fetal medido por ultrassonografia bidimensional como um meio de predizer a hipoplasia pulmonar (HP) neonatal, definida no momento do exame anatomopatológico ${ }^{6,12}$. Em 2006, Ruano et al. ${ }^{17}$ demonstrou que esta proporção pode ser também estimada utilizando a ultrassonografia tridimensional (US-3D) no lugar da ressonância nuclear magnética. No entanto, esta proporção nunca foi comparada com a relação dos volumes totais pulmonares, contralateral e ipsilateral em fetos com HDC isolada para predizer a evolução perinatal. Além disso, medidas dos volumes pulmonares nesses casos pela US-3D nunca foram correlacionados com diagnóstico neonatal de HP grave e hipertensão arterial pulmonar (HAP).

Portanto, o presente estudo tem como objetivo comparar os diferentes índices de volume pulmonar entre si e com os resultados perinatais (óbitos perinatais, HP e HAP).

\section{PACIENTES E MÉTODOS}

Realizou-se estudo observacional prospectivo em um centro de Medicina Fetal terciária entre janeiro de 2002 e maio de 2007. Todas as gestantes submeteram-se voluntariamente ao exame de US3D após serem informadas sobre o protocolo de estudo. O protocolo foi aprovado pela Comissão de Ética local. As gestantes foram informadas de que os resultados do estudo não seriam utilizados para modificar a conduta médica. Os perinatologistas, ultrassonografistas e cirurgiões encarregados não tiveram conhecimento dos resultados obtidos na presente pesquisa.

A ultrassonografia foi realizada em 55 gestantes cujos fetos apresentavam HDC isolada (50 à esquerda e 5 à direita). A idade gestacional no momento do diagnóstico variou de 16 a 35 semanas. As medições dos pulmões fetais foram realizadas uma vez em cada caso, entre 22 e 36 semanas de gestação. Em todos os casos, a idade gestacional foi estabelecida com base na data da última menstruação e por medidas ultrassonográficas do comprimento cabeça-nádegas feitas no primeiro trimestre de gestação. Todos os fetos com HDC foram submetidos a exames detalhados de ultrassom e amniocentese para cariotipagem fetal. Somente casos com cariótipo normal e sem outras malformações associadas foram incluídos no estudo (HDC isolada). Nenhuma intervenção pré-natal foi realizada nesta série. As investigações de diagnóstico pré-natal, cuidados obstétricos e pós-tratamento foram fornecidos em um único centro.

Avaliação do volume pulmonar fetal pela US$3 D$

A avaliação dos volumes dos pulmões fetais pela US-3D foi realizado pela técnica descrita anteriormente ${ }^{11,13,16,17,20}$, utilizando aparelho de ultrassom Voluson 730 (Kretztechnik, General Electric-, Zipf, Áustria), com transdutor de 4-8 MHz com varredura de volume tridimensional. Cada pulmão foi cuidadosamente identificado nos três planos da imagem multiplanar e medido pela técnica rotacional usando o software VOCAL ${ }^{\mathrm{TM}}$, que consiste em delinear os contornos do pulmão repetidas vezes após rodar sua imagem em $30^{\circ}$ ( 6 vezes). Os volumes do pulmão 
Takashi E, et al. Diferentes índices volumétricos dos pulmões de fetos com hérnia diafragmática.

ipsilateral (do mesmo lado da HDC) e contralateral (do lado oposto da HDC) foram automaticamente medidos quatro vezes pelo mesmo operador e a média dessas medições foi considerada (Figura 1). O total do volume do pulmão fetal foi então calculado pela soma dos volumes do pulmão ipsilateral e contralateral.

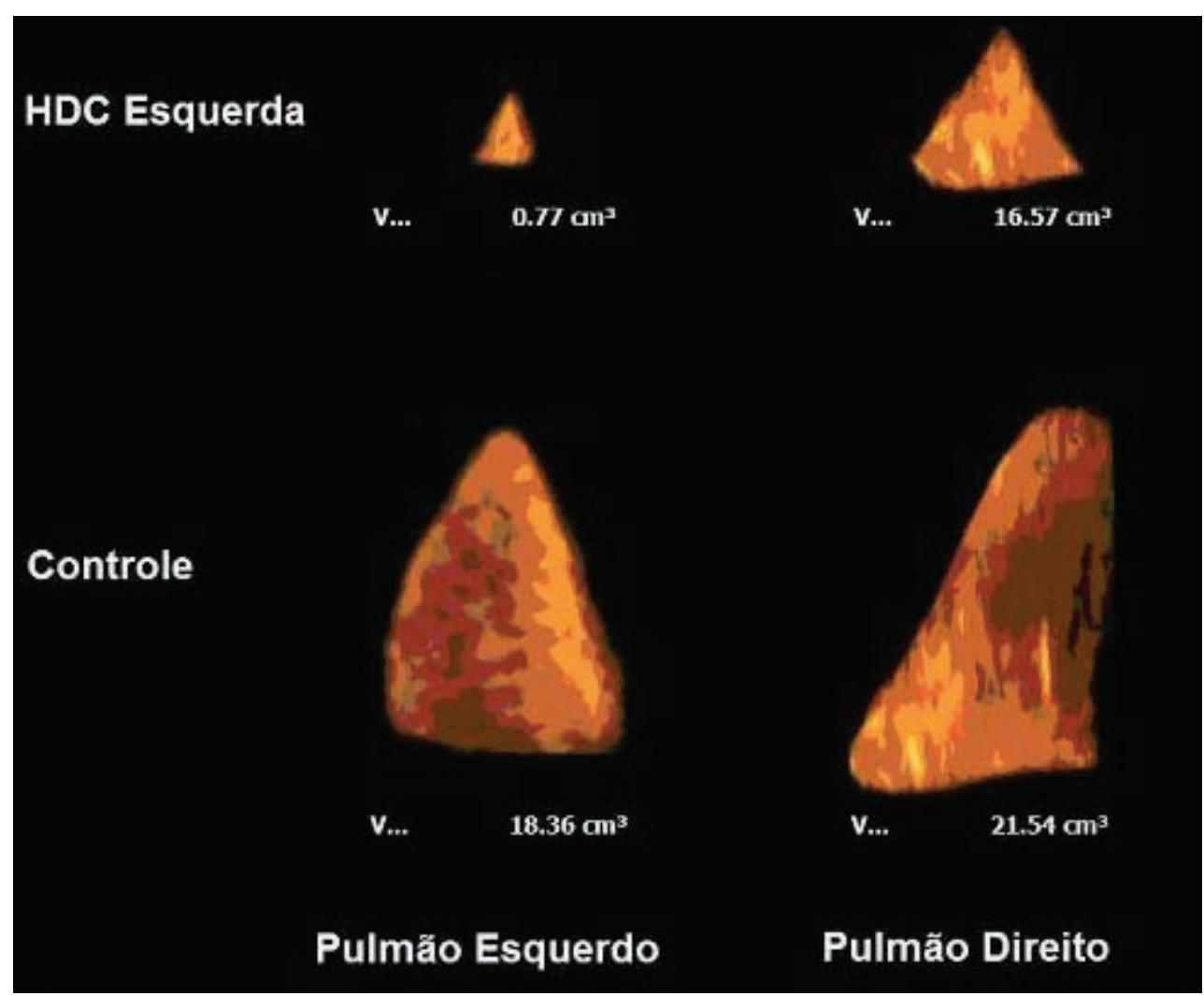

Figura 1. Imagem tridimensional dos pulmões direito e esquerdo de um feto normal (controle) e de outro com HDC, na $28^{\mathrm{a}}$ semana de gestação

2D

Estimativa do peso corporal fetal pela US-

Durante o mesmo exame, o peso corporal fetal foi estimado utilizando medições do diâmetro biparietal, circunferência cefálica, circunferência abdominal e comprimento do fêmur (equação de Hadlock) ${ }^{14}$.

\section{Conduta neonatal}

Imediatamente após o nascimento, os neonatos foram entubados e ventilação de alta frequência oscilatória foi iniciada em todos os casos, esta foi posteriormente alterada para a ventilação convencional quando apropriada. O óxido nítrico (NO) foi usada em lactentes apresentando hipertensão pulmonar. Reparação da HDC só foi realizada após estabilização hemodinâmica e respiratória préoperatória.
A HP grave foi considerada como um cenário clínico quando a baixa saturação de oxigênio (<80\%) foi persistente, enquanto o recém estava sendo mantido em 100\% FiO2 $\geq 48$ horas de vida. Além disso, nos casos que evoluíram para morte neonatal, uma razão pulmão / peso corporal $<0,012$ em idade gestacional $\geq 28$ semanas e $<0,015$ até $<28$ semanas no momento da autópsia foi utilizado para definir $\mathrm{HP}^{6,12}$. A HAP foi definida quando evidência ecocardiográfica de elevação de pressão pulmonar foi sentida por estar presente shunt direita-esquerda ou shunt bidirecional em todo o forame oval ou quando existia uma persistente diferença na fase de saturação pré- a pósductal com gradiente de oxigênio $>20 \%^{12,13}$.

\section{Análises estatísticas}

Os volumes dos pulmões totais, contralaterais e ipsilaterais foram comparados com curvas de referência descritas previamente ${ }^{15}$, e as relações dos 
volumes pulmonares observados/esperados totais (VPT), contralaterais observados/esperados (VPC) e ipsilaterais (VPI) foram então calculadas, assim como a relação entre volume pulmonar total e peso corpóreo fetal estimado (VP/PF). A correlação entre essas quatro relações foi calculada pelo coeficiente de correlação de Pearson. Essas relações de volumes pulmonares fetais foram analisadas de acordo com a evolução perinatal (óbitos perinatais, HP grave e HAP). Foram avaliadas também as precisões de VPT, VPC, VPI e VP/PF em predizer estas complicações perinatais pela curva ROC (SSPS14, Microsoft, E.U.A.). Considerou-se $p<0,05$ como diferença estatisticamente significante.

\section{RESULTADOS}

Trinta e uma $(56,4 \%)$ crianças evoluíram a óbito (30 óbitos neonatais e um óbito fetal). No grupo de morte neonatal, dois casos de HDC foram reparados.

A HP grave foi diagnosticado em 37 dos 55 casos $(67,3 \%)$ e confirmada pelo exame anatomopatológico em 28 dos 31 casos $(90,3 \%)$ que morreram. A HP grave estava presente em 10 dos 24 sobreviventes $(41,7 \%)$ e em 28 dos que morreram $(90,3 \%, p<0,01)$. A HAP foi observada clinicamente em 38 casos $(70,4 \%)$ com HDC, sendo diagnosticada em todos (100\%) que faleceram e em 10 dos $24(41,7 \%)$ sobreviventes $(p<0,01)$.

No grupo de morte neonatal $(n=30)$, vinte e oito bebês apresentaram tanto HP grave e HAP, enquanto dois apresentaram apenas HAP. Nos 24 casos que sobreviveram, 7 neonatos apresentaram ambos HP e HAP, dois casos apresentaram HP e apenas um foi tratado por HAP sem sinais de HP. HP grave e HAP ocorreram simultaneamente em 35 $(63,0 \%)$ de 54 crianças.

Dentre todos os fetos, valores medianos de VPT, VPC, VPI e VP/PF foram 0,37 (intervalo: 0,12-0,66), 0,53 (intervalo: 0,22-0,95), 0,21 (intervalo: 0,01-0,78) e 0.011 (intervalo: 0.004-0.022), respectivamente. O VPI (média: $0,21 \pm 0,22$ ) foi menor do que o VPC (média: 0,53 $\pm 0,21, \mathrm{p}<0,001$ ) (Figura 2). Correlações estatisticamente significantes foram observadas entre as quatro medidas de volume pulmonar fetal (Tabela 1).

Tabela 1. Correlações entre as diferentes medições de razões pulmonares por ultrassonografia tridimensional em HDC isolada

\begin{tabular}{lcc}
\hline & $\boldsymbol{r}$ & $\boldsymbol{p}$ \\
\hline VPT $x$ VPC & 0.89 & $<0.001$ \\
VPT $\times$ VPI & 0.82 & $<0.001$ \\
VPT $\times$ VP/PF & 0.89 & $<0.001$ \\
VPC $\times$ VPI & 0.53 & 0.08 \\
VPC $\times$ VP/PF & 0.91 & $<0.001$ \\
VPI x PF & 0.52 & 0.17 \\
\hline
\end{tabular}

VPT: observar a razão do volume pulmonar fetal total esperado VPC: observar a razão do volume pulmonar fetal contralateral esperado ; VPI: observar a razão do volume pulmonar fetal ipsilateral esperado; VP/PF: razão do volume pulmonar fetal ultrassonográfico pelo peso corporal.

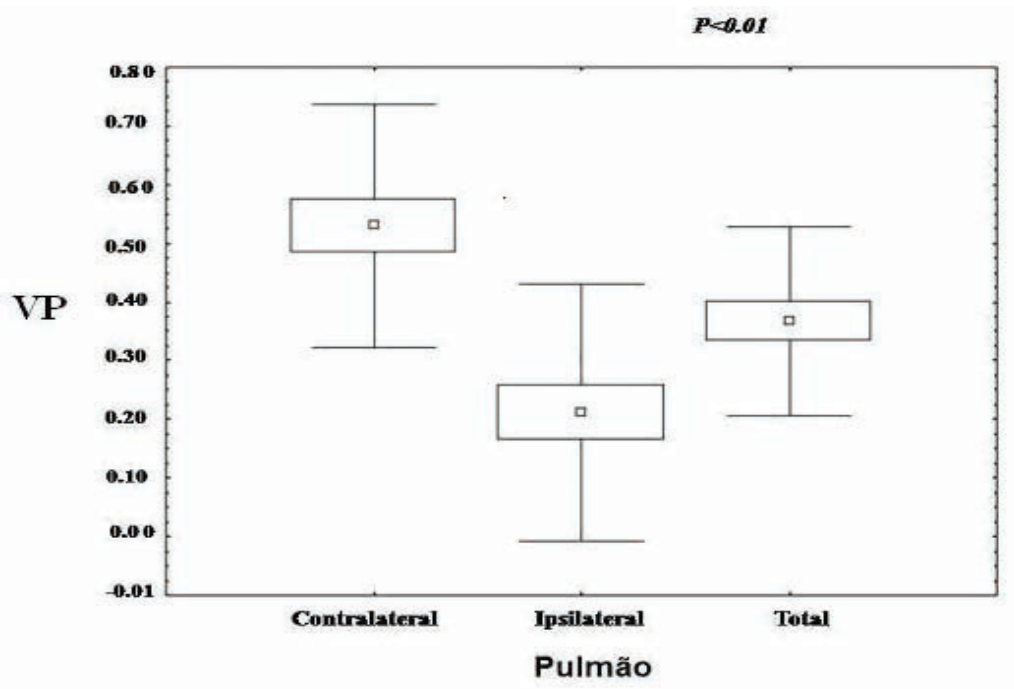

Figura 2. Gráfico da relação entre volumes pulmonares fetais observados/esperados considerando os pulmões contralaterais, ipsilateraisl e totais de fetos com HDC. A barra horizontal dentro da caixa corresponde ao valor médio. As barras superior e inferior das caixas correspondem à 1ํ e $3^{\circ}$ quartis (Q1 e Q3), respectivamente 
Takashi E, et al. Diferentes índices volumétricos dos pulmões de fetos com hérnia diafragmática.

Os VPT, VPC e VP/PF foram significativamente menores nos fetos que morreram em comparação com aqueles que sobreviveram (Tabela 2 ). As precisões de VPT, VPC e VP/PF em predizer óbito perinatal foram de $83,6 \%, 72,7 \%, 67,3 \%$ e $76,4 \%$, respectivamente (Tabela 3 ).

Os VPT, VPI e VP/PF foram significativamente menores nos fetos que apresentaram HP grave (Tabela 2). As precisões de VPT, VPI e VP/PF em predizer HP grave foram de $85,5 \%, 61,8 \%, 80,0 \%$ e $76,4 \%$, respectivamente (Tabela 4 ).

Apenas o VPT esteve associado estatisticamente significante com o diagnóstico pós-natal de HAP. A precisão do VPT em predizer a HAP foi de $81,5 \%$ (Tabela 5).

Tabela 2. Medições de pulmões avaliados no pré-natal por ultrassonografia tridimensional e o resultado perinatal em casos de hérnia diafragmática congênita isolada

\begin{tabular}{|c|c|c|c|c|c|c|c|c|c|}
\hline & \multicolumn{3}{|c|}{ Resultado perinatal } & \multicolumn{3}{|c|}{ Hipoplasia pulmonar grave } & \multicolumn{3}{|c|}{ Hipertensão Arterial Pulmonar* } \\
\hline & $\begin{array}{l}\text { Mortes Peri- } \\
\text { natal }(n=31)\end{array}$ & $\begin{array}{l}\text { Sobreviven- } \\
\text { tes }(n=24)\end{array}$ & $\boldsymbol{P}$ & $\begin{array}{l}\text { Presente } \\
(n=37)\end{array}$ & $\begin{array}{c}\text { Ausente } \\
(n=18)\end{array}$ & $p$ & Presente $(n=38)$ & Ausente $(n=16)$ & $p$ \\
\hline VPT & $\begin{array}{l}0.28 \\
(0.12-0.66)\end{array}$ & $\begin{array}{l}0.40 \\
(0.33-0.66)\end{array}$ & 0.001 & $\begin{array}{l}0.33 \\
(0.22-0.61)\end{array}$ & $\begin{array}{l}0.44 \\
(0.37-0.60)\end{array}$ & 0.01 & $\begin{array}{l}0.33 \\
(0.12-0.66)\end{array}$ & $\begin{array}{l}0.42 \\
(0.37-0.66)\end{array}$ & 0.001 \\
\hline VPC & $\begin{array}{l}0.38 \\
(0.22-0.94)\end{array}$ & $\begin{array}{l}0.59 \\
(0.52-0.92)\end{array}$ & 0.002 & $\begin{array}{l}0.52 \\
(0.22-0.68)\end{array}$ & $\begin{array}{l}0.55 \\
(0.52-0.95)\end{array}$ & 0.17 & $\begin{array}{l}0.53 \\
(0.22-0.95)\end{array}$ & $\begin{array}{l}0.54 \\
(0.52-0.92)\end{array}$ & 0.42 \\
\hline VPI & $\begin{array}{l}0.12 \\
(0.01-0.78)\end{array}$ & $\begin{array}{l}0.19 \\
(0.01-0.66)\end{array}$ & 0.39 & $\begin{array}{l}0.12 \\
(0.01-0.78)\end{array}$ & $\begin{array}{l}0.40 \\
(0.01-0.66)\end{array}$ & 0.04 & $\begin{array}{l}0.13 \\
(0.01-0.78)\end{array}$ & $\begin{array}{l}0.31 \\
(0.12-0.65)\end{array}$ & 0.05 \\
\hline $\begin{array}{l}\text { V P / } \\
\text { PF }\end{array}$ & $\begin{array}{l}0.007 \\
(0.004-0.022)\end{array}$ & $\begin{array}{l}0.011 \\
(0.010-0.021)\end{array}$ & 0.04 & $\begin{array}{l}0.009 \\
(0.004-0.012)\end{array}$ & $\begin{array}{l}0.015 \\
(0.011-0.22)\end{array}$ & 0.01 & $\begin{array}{l}0.010 \\
(0.003-0.022)\end{array}$ & $\begin{array}{l}0.011 \\
(0.011-0.21)\end{array}$ & 0.23 \\
\hline
\end{tabular}

VPT: observar a razão do volume pulmonar fetal total esperado; VPC: observar a razão do volume pulmonar fetal contralateral esperado; VPI: observar a razão do volume pulmonar fetal ipsilateral esperado; VP/PF: razão do volume pulmonar fetal ultrassonográfico pelo peso corporal. Os valores são relatados como mediana (intervalo). * Um caso foi excluído devido à morte do feto, no qual não foi possível avaliar clinicamente a presença de hipertensão arterial pulmonar.

Tabela 3. Previsão de morte perinatal na hérnia diafragmática congênita isolada utilizando diferentes medições de pulmões avaliados por ultrassonografia tridimensional

\begin{tabular}{lcccccc}
\hline Parâmetro & Média de corte & Sensibilidade & Especificidade & VPP & VPN & Precisão \\
\hline VPT & 0.35 & $80.6 \%(25 / 31)$ & $87.5 \%(21 / 24)$ & $89.3 \%(25 / 28)$ & $77.8 \%(21 / 27)$ & $83.6 \%(46 / 55)$ \\
VPC & 0.53 & $61.3 \%(19 / 31)$ & $87.5 \%(21 / 24)$ & $86.4 \%(19 / 22)$ & $63.6 \%(21 / 33)$ & $72.7 \%(40 / 55)$ \\
VPI & 0.16 & $74.2 \%(23 / 31)$ & $58.3 \%(14 / 24)$ & $69.7 \%(23 / 33)$ & $63.6 \%(14 / 22)$ & $67.3 \%(37 / 55)$ \\
VP/PF & 0.011 & $64.5 \%(20 / 31)$ & $91.7 \%(22 / 24)$ & $90.9 \%(20 / 22)$ & $66.7 \%(22 / 33)$ & $76.4 \%(42 / 55)$ \\
\hline
\end{tabular}

VPT: observar a razão do volume pulmonar fetal total esperado; VPC: observar a razão do volume pulmonar fetal contralateral esperado; VPI: observar a razão do volume pulmonar fetal ipsilateral esperado; VP/PF: razão do volume pulmonar fetal ultrassonográfico pelo peso corporal.

Tabela 4. Previsão de hipoplasia pulmonar grave em hérnia diafragmática congênita isolada utilizando diferentes medições de pulmões avaliados por ultrassonografia tridimensional

\begin{tabular}{lcllccc}
\hline Parâmetro & Média de corte & Sensibilidade & Especificidade & VPP & VPN & Acurácia \\
\hline VPT & 0.37 & $86.5 \%(32 / 37)$ & $83 / 3 \%(15 / 18)$ & $91.4 \%(32 / 35)$ & $75.0 \%(15 / 20)$ & $85.5 \%(47 / 55)$ \\
VPC & 0.53 & $51.4 \%(19 / 37)$ & $83.3 \%(15 / 18)$ & $86.4 \%(19 / 22)$ & $45.5 \%(15 / 33)$ & $61.8 \%(34 / 55)$ \\
VPI & 0.40 & $91.9 \%(34 / 37)$ & $55.6 \%(10 / 18)$ & $81.0 \%(34 / 42)$ & $76.9 \%(10 / 13)$ & $80.0 \%(44 / 55)$ \\
VP/PF & 0.012 & $78.4 \%(29 / 37)$ & $72.2 \%(13 / 18)$ & $85.3 \%(29 / 34)$ & $61.9 \%(13 / 21)$ & $76.4 \%(42 / 55)$ \\
\hline
\end{tabular}

VPT: observar a razão do volume pulmonar fetal total esperado; VPC: observar a razão do volume pulmonar fetal contralateral esperado; VPI: observar a razão do volume pulmonar fetal ipsilateral esperado; VP/PF: razão do volume pulmonar fetal ultrassonográfico pelo peso corporal. 
Tabela 5. Previsão pós-natal de hipertensão pulmonar arterial em hérnia diafragmática congênita isolada utilizando diferentes medições de pulmões avaliados por ultrassonografia tridimensional

\begin{tabular}{lllllll}
\hline Parâmetro & Média de corte & Sensibilidade & Especificidade & VPP & VPN & Precisão \\
\hline VPT & 0.39 & $78.9 \%(30 / 38)$ & $87.5 \%(14 / 16)$ & $93.8 \%(30 / 32)$ & $63.6 \%(14 / 22)$ & $81.5 \%(44 / 54)$ \\
VPC & 0.55 & $52.6 \%(20 / 38)$ & $50.0 \%(8 / 16)$ & $71.4 \%(20 / 28)$ & $30.8 \%(8 / 26)$ & $51.9 \%(28 / 54)$ \\
VPI & 0.40 & $84.2 \%(32 / 38)$ & $50.0 \%(8 / 16)$ & $80.0 \%(32 / 40)$ & $57.1 \%(8 / 14)$ & $74.0 \%(40 / 54)$ \\
VP/PF & 0.012 & $71.1 \%(27 / 38)$ & $50.0 \%(8 / 16)$ & $77.1 \%(27 / 35)$ & $42.1 \%(8 / 19)$ & $64.8 \%(35 / 54)$ \\
\hline
\end{tabular}

VPT: observar a razão do volume pulmonar fetal total esperado; VPC: observar a razão do volume pulmonar fetal contralateral esperado; VPI: observar a razão do volume pulmonar fetal ipsilateral esperado; VP/PF: razão do volume pulmonar fetal ultrassonográfico pelo peso corporal.

Um caso foi excluído devido a morte do feto, no qual não foi possível avaliar clinicamente a presença de hipertensão arterial pulmonar.

\section{DISCUSSÃO}

O presente estudo confirma que a HDC está associada a uma elevada taxa de mortalidade perinatal, o que está relacionado com a gravidade da HP e / ou HAP. É bem aceito que a principal causa de morte perinatal em HDC isolada é secundária a $\mathrm{PH}$ grave, no entanto, o presente estudo destaca que a mortalidade perinatal também ocorre devido à HAP grave neonatal. Esses dois distúrbios geralmente ocorrem simultaneamente na grande maioria dos casos de HDC. No entanto, achados clínicos apóiam na hipótese de que, em alguns casos, a HP é a doença predominante acompanhada somente de ligeira a moderada HAP. Em outros casos, a doença predominante é HAP grave com HP leve. Os resultados presentes parecem concordar com este fato. HP grave esteve presente em 27 $(87,1 \%)$ crianças que morreram, enquanto HAP esteve presente em todas (100\%) elas. Três bebês apresentaram HAP sem HP grave, o que significa que estes pacientes apresentavam pulmões maiores (volumes) com vascularização pulmonar anormal.

Em contrapartida, a HP grave esteve presente em 10 dos $24(41,7 \%)$ das crianças que sobreviveram, sete delas também apresentando HAP. Duas crianças que sobreviveram apresentaram hipoplasia pulmonar sem qualquer sinal de HAP.

Considerando todas as crianças, a HP grave e a HAP ocorreram simultaneamente em 35 (63,0\%) dos 54 pacientes. Isso pode explicar a razão pela qual medidas dos tamanhos dos pulmões fetais pode apresentar precisão baixa em predizer óbitos perinatais em casos com HDC isoladas. O estudo de outros parâmetros pré-natal que avalia a função pulmonar ou o estado vascular fetal, em tais casos, ainda é necessário. É possível que os índices vasculares avaliados por ultrassonografia 3D-power
Doppler pode predizer melhor a precisão, uma vez que avalia a função pulmonar e de HAP ${ }^{11}$.

Tal como referido anteriormente, nos exames patológicos de neonatos que morrem de HDC isolada, o pulmão ipsilateral é geralmente menor do que o pulmão contralateral. Estes resultados foram confirmados no presente estudo, uma vez que VPI foi significativamente menor do que VPC. Esta diferença pode também ajudar a explicar a variável da capacidade das proporções dos pulmões estudados para predizer o resultado perinatal.

A grande vantagem de avaliar todas estas razões não é apenas estabelecer qual é a maneira mais precisa em predizer a morte perinatal, mas também para melhor compreensão dos processos fisiopatológicos envolvidos na HDC isolada. Por exemplo, quando se considera o pulmão contralateral isoladamente, a predição de mortes perinatais é possível, mas não de HP e/ou HAP, uma vez que este pulmão não é tão gravemente afetado (hipoplásico). Em contrapartida, considerando apenas o pulmão ipsilateral, é possível predizer a HP grave, mas não os óbitos perinatais e/ou HAP, porque esse pulmão é tão gravemente afetado (hipoplásico) que se torna difícil determinar diferentes valores entre fetos que evoluíram ao óbito dos que sobrevivem. Além disso, nos pulmões ipsilaterais, os vasos do parênquima pulmonar estão tão seriamente comprometidos, o que se torna também impossível diferenciar o estado de desenvolvimento vascular pulmonar. Avaliar o volume pulmonar fetal total melhora estes resultados, uma vez que este parâmetro considera ambos os pulmões em conjunto.

No presente estudo, o propósito de avaliar a relação dos volumes pulmonares foi confirmado. A comparação das medidas dos pulmões fetais observados com os esperados ajudou a ajustar os dados específicos para a idade gestacional. Alguns 
Takashi E, et al. Diferentes índices volumétricos dos pulmões de fetos com hérnia diafragmática.

pesquisadores acreditam que a proporção relativa do pulmão poderia ser inviável para analisar fetos apresentando restrição de crescimento. No entanto, é importante mencionar que curvas de normalidade consideram este fato, uma vez que fetos normais que apresentam restrição de crescimento não devem ser excluídos dos dados utilizados para a construção dessas curvas $^{15}$. Mesmo assim, o VP/PF também foi avaliado neste trabalho e, embora não seja considerado um valor de referência específica para a idade gestacional, este levou em conta o peso fetal. Comparando a proporção relativa do pulmão (VPT) com o VP/PF, a primeira mostrou maior eficácia do que a segunda. A principal vantagem do VPT é que este fornece comparação com o pulmão normal, quantificando o volume total reduzido. O VP/PF pode ser uma boa razão para determinar se o feto apresenta hipoplasia pulmonar, mas não é bom o suficiente para quantificar a sua gravidade, uma vez que esta razão é expressa em valores muito pequenos.

Em conclusão, VPT é a melhor relação das medidas dos pulmões fetais pelo US-3D para predizer os óbitos perinatais, a HP grave e a HAP, entre aqueles. Mesmo assim, é possível predizer apenas $83,6 \%$ dos óbitos perinatais utilizando a tecnologia de US-3D, o que significa que há a necessidade de se estudar outros métodos pré-natais possivelmente que avaliem a função e/ou a vascular dos pulmões fetais em fetos com HDC isolada.

Takashi E, Ruano R, Zugaib M. Different volumetric indices of lungs in fetuses with congenital diaphragmatic hernia by three-dimensional ultrasonography. Rev Med (São Paulo). 2009 jan.-mar.;88(1):58-65.

\begin{abstract}
Objective: To evaluate the potential of different lung measurements using threedimensional ultrasonography (3DUS) to predict perinatal outcome in cases of isolated congenital diaphragmatic hernia (CDH). Study design: Fifty-five fetuses presenting isolated $\mathrm{CDH}$ were prospectively evaluated by 3DUS between January 2002 and May 2007. Observed/expected total, contralateral and ipsilateral fetal lung volume ratios TFLV, CFLV and IFLV, respectively) were calculated using the VOCAL ${ }^{\mathrm{TM}}$ technique and ultrassonographic fetal total lung volume to body weight ratio (US-FLW). These lung measurements were compared to one another and to perinatal outcomes: perinatal deaths, severe pulmonary hypoplasia $(\mathrm{PH})$ and pulmonary arterial hypertension (PAH). Results: Perinatal death occurred in 31 of 55 infants (56.4\%), severe $\mathrm{PH}$ in 37 of 55 infants $(67.3 \%$ ) and $\mathrm{PAH}$ in 38 of 54 (excluding one death in utero) of neonates (70.4\%). Severe PH and PAH occurred simultaneously in 35 (63.0\%) of 54 infants. Good correlations between all lung volumetric ratios were observed. O/e-TotFLV, o/e-ContFLV and US-FLW correlated statistically with perinatal deaths; o/e-TotFLV, o/e-IpsiFLV and USFLW with postnatal diagnosis of severe $\mathrm{PH}$; while only o/e-TotFLH correlated statistically with postnatal diagnosis of PAH. The accuracies of o/e-TotFLV, o/e-ContFLV, o/e-IpsiFLV and US-FLW in predicting perinatal deaths were $83.6 \%, 72.7 \%, 67.3 \%$ and $76.4 \%$, respectively. Conclusion: O/e-TotFLV using 3D-US appeared to be the most accurate predictor of perinatal deaths as it can predict both $\mathrm{PH}$ and $\mathrm{PAH}$.
\end{abstract}

KEY WORDS: Hernia diaphragmatic/congenital. Imaging three-dimensional. Prenatal diagnosis. Lung/abnormalities. Ultrasonography, prenatal. Lung diseases/congenital.

\title{
REFERÊNCIAS
}

1. Askenazi SS, Perlman M. Pulmonary hypoplasia: lung weight and radial alveolar count as criteria of diagnosis. Arch Dis Child. 1979;54:614-8.

2. Bahlmann F, Merz E, Hallermann C, Stopfkuchen H, Kramer W, Hofmann M. Congenital diaphragmatic hernia: ultrasonic measurement of fetal lungs to predict pulmonary hypoplasia. Ultrasound Obstet Gynecol. 1999;14:162-8.

3. Deprest J, Gratacos E, Nicolaides KH. FETO Task Group. Fetoscopic tracheal occlusion (FETO) for severe congenital diaphragmatic hernia: evolution of a technique and preliminary results. Ultrasound Obstet Gynecol. 2004;24:121-6.

4. Greenough A, Khetriwal B. Pulmonary hypertension in the newborn. Paediatr Respir Rev. 2005;6:111-6.

5. Hadlock FP, Harrist RB, Sharman RS, Deter RL, Park SK. Estimation of fetal weight with the use of head, body, and femur measurements--a prospective study. Am J Obstet Gynecol. 1985;151:333-7

6. Harrison MR, Keller RL, Hawgood SB, Kitterman JA, Sandberg PL, Farmer DL, et al. A randomized 
trial of fetal endoscopic tracheal occlusion for severe fetal congenital diaphragmatic hernia. N Engl J Med. 2003;349:1916-24.

7. Laurita MR, Gonik B, Romero R. Pulmonary hypoplasia: pathogenesis, diagnosis, and antenatal prediction. Obstet Gynecol. 1995;86:466-75.

8. Lipshutz GS, Albanese CT, Feldstein VA, Jennings RW, Housley HT, Beech R, et al. Prospective analysis of lung-to-head ratio predicts survival for patients with prenatally diagnosed congenital diaphragmatic hernia. J Pediatr Surg. 1997;32:1634-6.

9. Mahieu-Caputo D, Sonigo $P$, Dommergues $M$, Fournet JC, Thalabard JC, Abarca C, et al. Fetal lung volume measurement by magnetic resonance imaging in congenital diaphragmatic hernia. BJOG. 2001;108:863-8.

10. Metkus AP, Filly RA, Stringer MD, Harrison MR, Adzick NS. Sonographic predictors of survival in fetal diaphragmatic hernia. J Pediatr Surg. 1996;31:148-51.

11. Roofthooft MT, Bergman KA, Waterbolk TW, Ebels $\mathrm{T}$, Bartelds B, Berger RM. Persistent pulmonary hypertension of the newborn with transposition of the great arteries. Ann Thorac Surg. 2007;83:1446-50.

12. Ruano R, Aubry MC, Barthe B, Mitanchez D, Dumez Y, Benachi A. Quantitative analysis of fetal pulmonary vasculature by 3-dimensional power Doppler ultrasonography in isolated congenital diaphragmatic hernia. Am J Obstet Gynecol. 2006;195:1720-8.

13. Ruano R, Benachi A, Joubin L, Aubry MC, Thalabard JC, Dumez Y, et al. Three-dimensional ultrasonographic assessment of fetal lung volume as prognostic factor in isolated congenital diaphragmatic hernia. BJOG. 2004;11:423-9.
14. Ruano R, Benachi A, Martinovic J, Grebille AG, Aubry MC, Dumez Y, et al. Can three-dimensional ultrasound be used for the assessment of the fetal lung volume in cases of congenital diaphragmatic hernia? Fetal Diagn Ther. 2004;19:87-91.

15. Ruano R, Joubin L, Aubry MC, Thalabard JC, Dommergues $\mathrm{M}$, Dumez $\mathrm{Y}$, et al. A nomogram of fetal lung volumes estimated by 3-dimensional ultrasonography using the rotational technique (virtual organ computeraided analysis). J Ultrasound Med. 2006;25:701-9.

16. Ruano R, Joubin L, Sonigo P, Benachi A, Aubry MC, Thalabard JC, et al. Fetal lung volume estimated by 3-dimensional ultrasonography and magnetic resonance imaging in cases with isolated congenital diaphragmatic hernia. J Ultrasound Med. 2004;23:353-8.

17. Ruano R, Martinovic J, Aubry MC, Dumez Y, Benachi A. Predicting pulmonary hypoplasia using the sonographic fetal lung volume to body weight ratio--how precise and accurate is it? Ultrasound Obstet Gynecol. 2006;28:958-62.

18. Ruano R, Martinovic J, Dommergues M, Aubry MC, Dumez Y, Benachi A. Accuracy of fetal lung volume assessed by three-dimensional sonography. Ultrasound Obstet Gynecol. 2005;26:725-30.

19. Tanigaki S, Miyakoshi K, Tanaka M, Hattori Y, Matsumoto T, Uno K, et al. Pulmonary hypoplasia: prediction with use of ratio of MR imaging-measured fetal lung volume to US-estimated fetal body weight. Radiology. 2004;232:767-72.

20. Walsh-Sukys MC, Tyson JE, Wright LL, Bauer CR, Korones SB, Stevenson DK, et al. Persistent pulmonary hypertension of the newborn in the era before nitric oxide: practice variation and outcomes. Pediatrics. 2000;105:14-20. 